\title{
Artificial Immune Networks: Models and Applications
}

\author{
Xian Shen ${ }^{1}$, X. Z. Gao ${ }^{2,3}$, and Rongfang Bie ${ }^{3 *}$ \\ ${ }^{1}$ College of Information Science and Technology \\ Beijing Normal University, Beijing 100875, P. R. China \\ shenxian8307@gmail.com \\ ${ }^{2}$ Department of Electrical Engineering, Helsinki University of Technology \\ Otakaari 5 A, FI-02150 Espoo, Finland \\ ${ }^{3}$ College of Information Science and Technology \\ Beijing Normal University, Beijing 100875, P. R. China \\ gao@cc.hut.fi \\ ${ }^{3}$ College of Information Science and Technology \\ Beijing Normal University, Beijing 100875, P. R. China \\ rfbie@bnu.edu.cn \\ Received:20-08-2007 Revised:07-12-2007
}

\begin{abstract}
Artificial Immune Systems (AIS), which is inspired by the nature immune system, has been applied for solving complex computational problems in classification, pattern recognition, and optimization. In this paper, the theory of the natural immune system is first briefly introduced. Next, we compare some well-known AIS and their applications. Several representative artificial immune networks models are also discussed. Moreover, we demonstrate the applications of artificial immune networks in various engineering fields.
\end{abstract}

Keywords: natural immune systems, artificial immune systems, artificial immune networks, learning algorithm, data mining.

\section{Introduction}

An immune system, one of the most intricate biological systems, has been used as a metaphor for intelligent computation in a variety of domains. Artificial Immune System (AIS) has been considered as a family of techniques originated from the community of immunology. As an important constituent of the AIS, the artificial immune networks are based on the principles of the behaviors of both $B$ cells and $T$ cells in the biological immune system. B cell is an integral part of the immune system. Through a process of recognition and stimulation, the B cells can clone and mutate to produce a diverse set of antibodies in an attempt to remove the infection from the body. T cell has two types. One regulates and controls the strength of the immune response, and the other directly destroys the cells that have specific antigens. Both the $B$ cells and $T$ cells have been widely employed to solve a wide range of engineering problems, such as anomaly detection and data mining. This paper aims at giving a concise overview on the artificial immune network models including their theory, structures, and applications.

The remainder of our paper is organized as follows. Section 2 provides a brief survey of relevant immunology. Section 3 discusses several typical models of artificial immune networks. The applications of these artificial immune networks in data mining, associative classification, pattern recognition, and function optimization are presented in Section 4. Finally, Section 5 concludes this paper with some remarks and conclusions.

\section{Natural Immune System}

A natural immune system has a very complex 'hunt and destroy' mechanism working on the cellular level inside our bodies [1]. It has the remarkable ability of learning about foreign substances (pathogens) that enter the body and responding to them by producing the antibodies, which can attack the antigens associated with the pathogens [2]. Generally, the human immune system consists of the antigen-presenting cells, lymphocytes, and antibodies. The lymphocytes are a kind of white blood cells, or more specifically, $\mathrm{B}$ lymphocytes and $\mathrm{T}$ lymphocytes [3]. These cells aid in recognizing and destroying specific substances. A B cell contains the antibodies on its surface, which can recognize the antigens invading the human body. The regions on the molecules the paratope can attach to are called epitopes. Identification of the antigen is achieved by the complementary matching between their paratope and epitope, comparable to 'lock and key' [4]. Figure 1 illustrates how an antigen-presenting cell processes and presents an antigen. T cells can interact with the antigen under the help of the antigen presenting and major histocompatibility complex molecules. More precisely, (1) in Fig. 1 illustrates that once an Antigen Presenting Cell (APC) finds the antigen, it internalizes the antigen, and fragments it into antigenic peptides. In (2), pieces of these peptides are incorporated into the MHC molecules, and in (3), these peptidesa are displayed on the surface of the cell. The $\mathrm{T}$ cell recognizes and interacts with this peptide-MHC molecule complex using its own receptor. Note that the $\mathrm{T}$ cells do not interact with the antigen directly. Instead they attach to the cell displaying foreign antigen complexes on the basis of an MHC molecule [3]. 


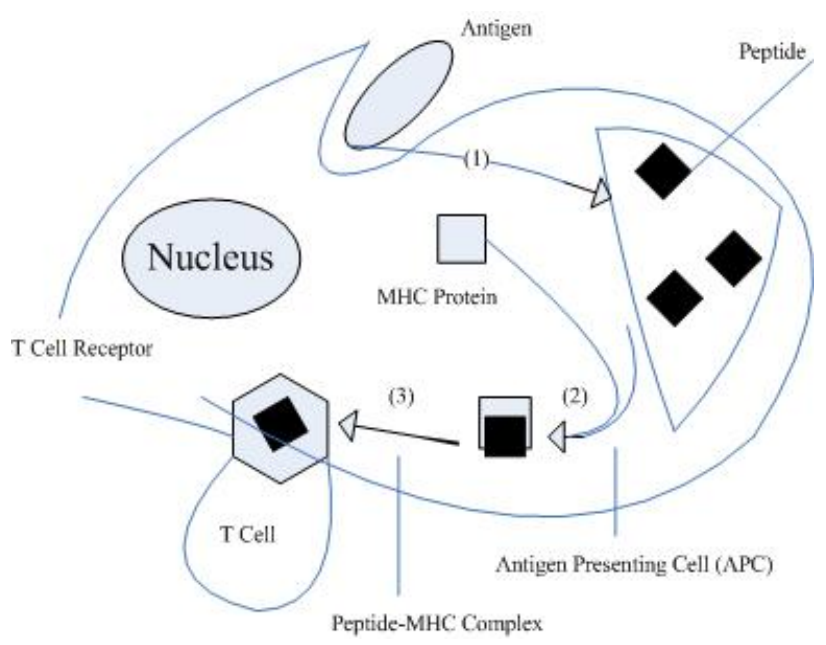

Figure 1. Processing and presentation of antigen

An antibody recognizes and eliminates only a specific type of antigens. The key portion of the antigen recognized by the antibody is called epitope, an antigen determinant, as shown in Figure 2. The paratope is the portion of the antibody that corresponds to a certain kind of antigens. When an antibody merges an antigen via the epitope and paratope, it can attack this antigen [3].

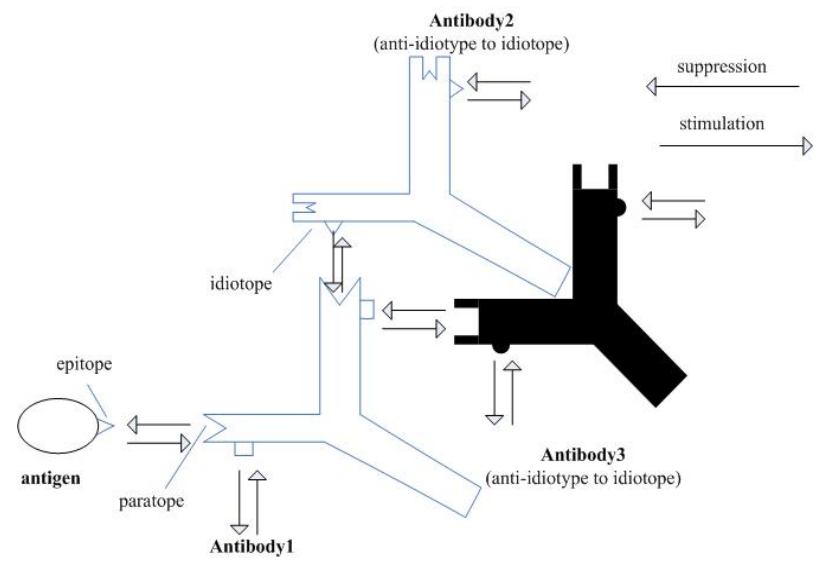

Figure 2. Interactions between antibody and antigen in natural immune system

The human immune system maintains a memory of infections so that if ever exposed to the same antigen, a quicker response can be elicited against the infection. In other words, the secondary immune response occurs, when the same antigen is indeed encountered. It is characterized by a rapider and more abundant production of the antibody, which results from the primary response [5].

\section{Typical Artificial Immune Networks}

In this section, a few representative artificial immune network models are introduced. We will discuss these models' theory, structures, and learning algorithms in details.

\section{A. Resource Limited Artificial Immune System}

The Resource Limited Artificial Immune System (RLAIS) [2] is built upon the work of the basic AIS [4]. As we know, the AIS consist of a set of B cells, links among themselves via their stimulation levels, and cloning and mutation operations performed on these B cells. The AIS can cluster together similar patterns in the training data presented. This network of $\mathrm{B}$ cells represents clusters with the affinity links [4]. However, several critical parameters should be manually set for efficient initialization: number of iterations, the Network Affinity Threshold (NAT), and mutation rate [5], which are explained as follows [5].

1. The number of iterations that the training data are presented to a learning system is not straightforward to choose. There is no direct correlation between the number of iterations and linkage value. Nevertheless, some experiences-based observations are clear: a). the more times the training set is presented to the artificial immune network, the longer it takes for the network to converge. In fact, there is an exponential growth in the network size, regardless of the training set, mutation rate, and NAT. b). We do not have a definite border line between presenting the training data in an insufficient or excessive number of times. Too many presentations of the training data may lead to a large network that is difficult interpret. The appropriate selection of this initialization parameter is clearly vital in the successful training of the AIS. Figure 3 of [5] tracks the evolution procedure of the RLAIS with the Iris data set as the training samples. Figure 3 (a) shows the resulting network after two iterations. It clearly demonstrates one separate cluster from the main cluster. Figure 3 (b) illustrates the network after five iterations, from which it is nearly impossible to observe any network structure.

2. The NAT is defined in (1), which is calculated by parsing the training data and finding the average Euclidean distance between each item. In (1), $1_{\mathrm{i}}$ represents the affinity associated with the ith link in the network, $\mathrm{nl}$ is the number of links present, and $\mathrm{A}$ is a constant value, where $0 \leq \mathrm{A} \leq 1$.

$$
\mathrm{NAT}=\mathrm{A} \frac{\left\{\sum_{\mathrm{i}=0}^{\mathrm{nl}} \operatorname{aff}\left(\mathrm{l}_{\mathrm{i}}\right)\right\}}{\mathrm{nl}}
$$

If the affinity between two B cells is less than the NAT, a link is created between them. As a matter of fact, the NAT directly influences the network linkage, and it should be chosen as a small value, e.g., 0.1, which can decrease the potential connectivity of the AIS, and, thus, separate out the training data more efficiently than if it has larger values.

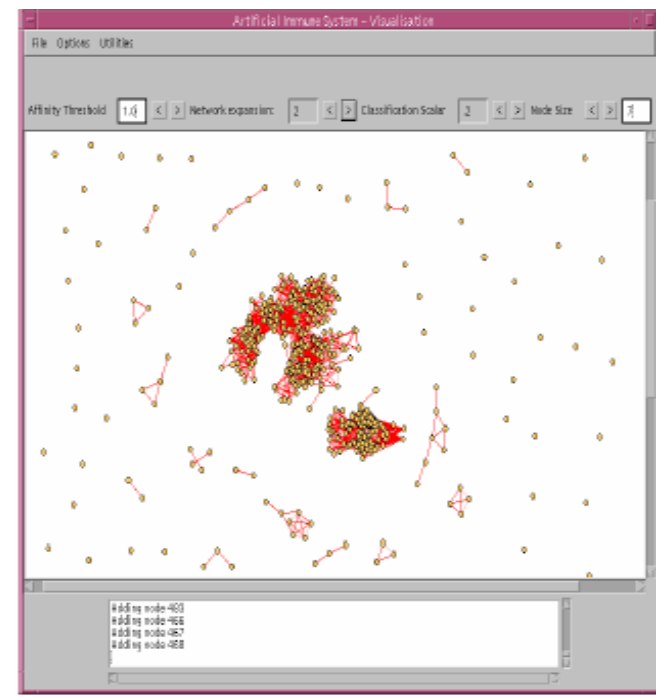

(a) after two iterations. 


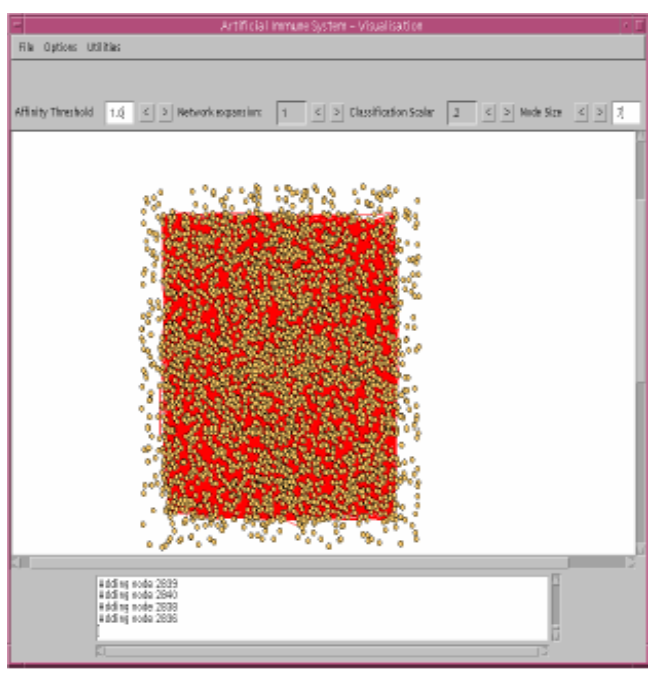

(b) after five iterations.

Figure 3. RLAIS-based clustering of Iris data set

3. Since the mutation rate creates a diverse representation of the training data, it can reduce the connectivity in the AIS. If the mutation rate is higher, it will produce a network with fewer B cells. Figure 4 shows the growth of the network linkage over training time with a mutation rate of $10 \%$ on the Iris data set.

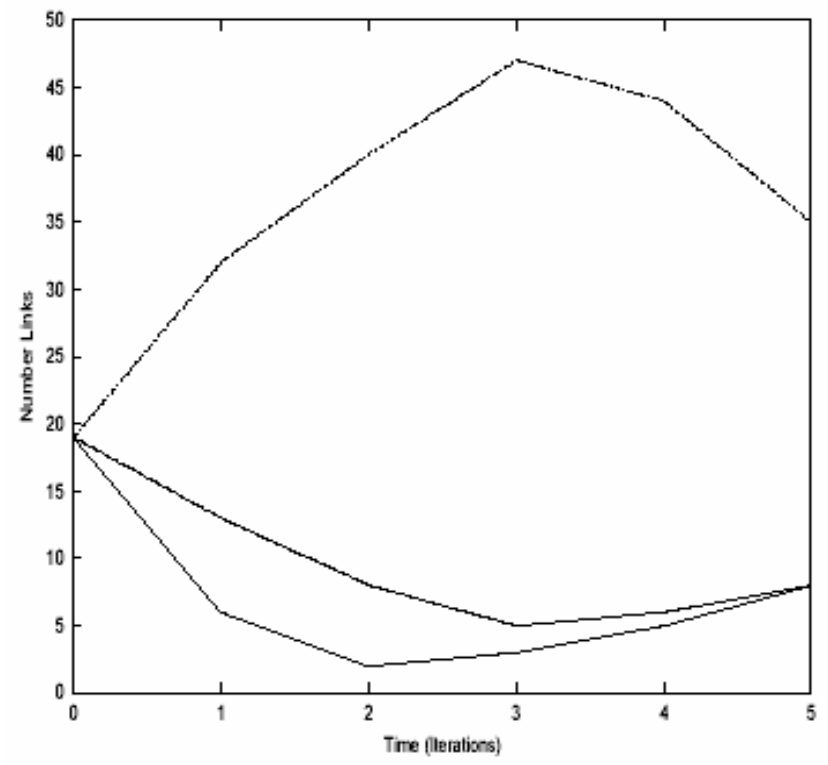

Figure 4. Linkage growth with mutation rate of $10 \%$ on Iris data set

The main difference between an AIS and a RLAIS is the removal of the number of times the training data set is presented to the network [5]. The RLAIS does not require setting the number of training cycles beforehand, but can still offer a more effective population control strategy. These improvements are due to the deployment of the Artificial Recognition Ball (ARB). The ARB is a representation of a certain number of identical B cells, because individual B cells are no longer explicitly represented in the RLAIS. The RLAIS has a group of ARBs as well as links among them. All the ARBs compete for representing the B cells within the RLAIS on the basis of their stimulation levels. The higher the ARB stimulation level, the more B cells it can represent. Once an ARB does not represent any B cells, it is expunged from the network. Indeed, the goal of the mutation mechanism is to create new ARBs that can better fit the training data. As new ARBs are created, some of them are satisfactory matching of the training data, while others are not. The latter are less stimulated by the training data and network, and will be ultimately removed from the RLAIS. The 'base' size of the network is obtained after a number of training iterations. Thus, over-training is not a serious problem in the RLAIS. The explosion in population growth can be eliminated by the above new population control scheme. Clearly, compared with the AIS population growth, no exponential growth exists in the RLAIS. Additionally, instead of recalculating the NAT at the end of each training epoch in the AIS, we keep it constant throughout the whole learning process in the RLAIS.

The RLAIS can be also used as a solid basis for continuous learning: previously unseen input data is presented to the RLAIS to allow new patterns to be learnt without adversely affecting those patterns already learnt. Unfortunately, there are a few unsolved problems in designing our RLAIS. For example, choosing the number of B cells that the RLAIS should allocate is an important factor. If there are too many resources selected, the network will become too large and unrepresentative, while too few resources can result in some un-captured patterns. Reference [6] proposes a new Self-Stabilizing Artificial Immune System (SSAIS) to deal with this drawback. The SSAIS differs from the RLAIS in that there is no fixed quantity of resources to be centrally distributed among the ARBs. The concept of resources is still used, but in an altered way. In the SSAIS, the resources are handled locally by each ARB. The ARB can increase its own resource allocation, when it registers the highest stimulation for an incoming data sample [6].

\section{B. aiNet}

The aiNet is an emerging kind of the AIS inspired by the immune network theory firstly proposed by Jerne in 1974 [7]. It is developed based on the ideas and concepts of three theories: the immuned network theory, the clonal selection principle, and affinity maturation principle [21]. The main role of this artificial immune network is to perform data compression by following the clonal selection as well as affinity maturation principles. The immune network theory hypothesizes the activities of the immune cells, emergence of memory, and discrimination between our own cells (known as self) and external invaders (known as non-self). The aiNet model consists of a group of cells, namely antibodies, interconnected by links with associated connection strengths. The aiNet antibodies represent the network internal images of the pathogens (input patterns), to which they are exposed. The connections among these antibodies determine their interrelations with providing a degree of similarity among themselves in the given metric space. The closer the antibodies, the more similar they are. This approach results in an antibody network that can recognize the antigens (input data set) with an adjustable generality.

The aiNet learning procedure can be divided in two principal steps [7]. The first one corresponds to the clonal selection principle and affinity maturation interactions, where the antibodies $(\mathrm{Ab})$ suffer from the cloning and mutation in order to recognize the antigens (Ag). This stage is actually similar to the CLONal selection ALGorithm (CLONALG) originally 
proposed by de Castro and von Zuben. The raw training data set is explored and compressed by the aiNet leading to an antibody network that extracts the most relevant information from the data for the clustering purposes. The second step of the aiNet includes the immune network interactions and introduction of diversity. The Minimal Spanning Tree (MST) is built on the antibody network, and its inconsistent edges are identified and removed, which can transform the network (data) separation into clusters.

In general, the aiNet is developed to answer the following important questions: is there a great amount of redundancy within the training data set, and, if there is, how can we reduce it? Are there any subgroups intrinsic to the data? How many groups are there in the input data set? What is the structure of the spatial distribution of these data? How can we generate decision rules to classify novel samples [8]? However, the main drawbacks of the aiNet, as pointed out in [9], are the large number of application dependent parameters and processing overhead of each iteration.

The aiNet can be considered as an evolutionary artificial immune network, because the evolution strategies based on the genetic variation and selection within a population of individuals are used to control the network dynamics and plasticity. Reference [10] proposes that the aiNet is capable of detecting the optimal solutions. Combined with the constant modulus criterion, it has been employed for the optimal blind IIR equalization. Furthermore, the aiNet is also a connectionist system, in which a matrix of connection strengths is defined to measure the affinities among the network cells. The learning algorithm targets at building a memory set that can recognize and represent the structural organization of the training data. Especially, the suppression threshold controls the specificity level of the cells, clustering accuracy, as well as network plasticity.

\section{C. iNet}

The iNet is a general framework for simulating the natural immune networks and further describing how to develop the AIS [3]. It utilizes the essential principles of the AIS, such as autonomous policy negotiation and system reconfiguration facility, for communication. The iNet serves as an information infrastructure, and can be applied to explore related mechanisms. Additionally, it helps to investigate a family of applications based on the artificial immune networks. As we know, application frameworks and patterns can enhance the reuse techniques, reduce development cost, and improve the quality of applications. The reusable components within the iNet are designed with several software patterns. Therefore, the iNet can explicitly show the developers its design intents and extension points, with which they can effectively tailor their own applications. More precisely, it is designed to allow the proper customization of various strategies to construct the artificial immune networks, e.g., network topology and network dynamics control. The iNet contains four main packages: GUI visualization, graph management, immuno-component management, and persistence \& exchange, as illustrated in Figure 5.

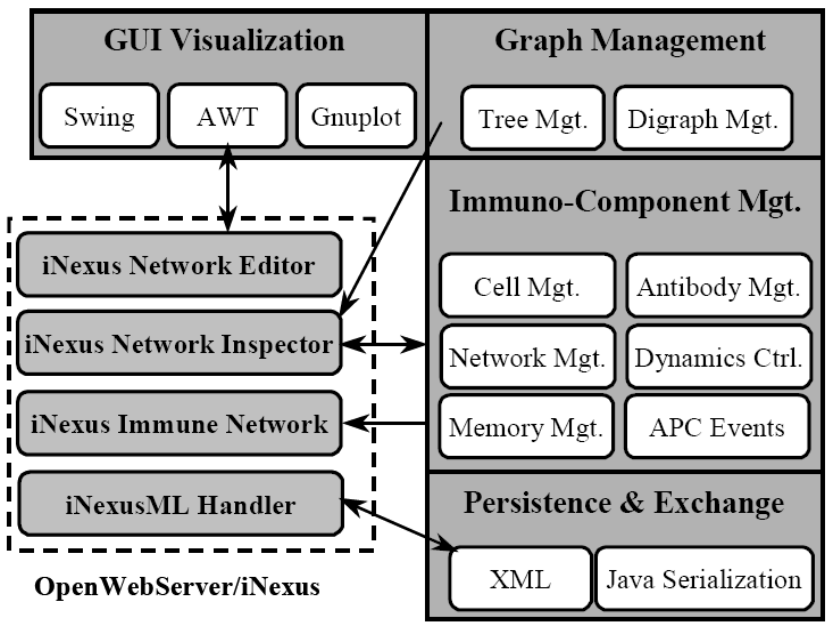

Figure 5. Structure of iNet.

D. IPAisys

The choice of an AIS model, including its expression, matching, training, evaluation, and various controlling parameters, is in general pre-determined by experts based on their experience on hypothesized problem space. The model is next iteratively adjusted until satisfactory outcome has been achieved in both training and testing. If there is no or few improvement during this process, one needs to resort to other models. Due to the limited experience of individual experts, it is nontrivial to define a robust AIS model for detecting all the possible objects.

Z. Wu and Y. Liang [26] propose an Integrated Platform of Artificial Immune Systems (IPAisys) based on the detector population mode to tackle the aforementioned problems. In this platform, a group of model prototypes are integrated in a unified framework, wherein the model structure can be automatically regularized by using the output of training and testing, as so-called "pressure". A self-regulating algorithm is developed with the use of model library to achieve the optimal multi-AIS-models through dynamic configuration. Therefore, this platform is flexible and intelligent, because it is capable of choosing an optimal combination of different models to cooperatively evaluate each unknown case.

In recent years, there have been numerous AIS models proposed for different intended applications. A. Iqbal and $\mathrm{M}$. A. Maarof [34] suggest an artificial APC model for danger susceptible data codons. S. Stepney, R. E. Smith, J. Timmis et al [35] present that the bio-inspired algorithms are best developed and analyzed in the context of a multidisciplinary conceptual framework that provides for sophisticated biological models and well-founded analytical principles. A framework in the context of the AIS network models is also outlined in [35].

\section{Applications of Artificial Immune Net- works}

During the past decade, the artificial immune networks have been successfully applied to a large variety of engineering areas, such as data mining [32], time series prediction, pattern recognition [33], optimization, fault detection [30], computer security [31], and process control. In this section, a few examples of these applications are demonstrated and discussed. We will also present our work on the clustering applications of the AIS. It illustrates that our AIS-based clustering algo- 
rithm is better than the commonly used clustering method, K-means [39].

\section{A. Data mining}

The Artificial Immune Network (AINE) is a kind of the AIS developed for data analysis and pattern discovery [13]. It employs some high-level metaphors drawn from the natural immune system, e.g., B cells are capable of recognizing pathogens (antigenic recognition), similar B cells are linked together and these links form a network of B cells (immune network theory), cloning and mutation operations are performed on B cells (clonal selection and somatic hypermutation), and a certain number of $\mathrm{B}$ cells can be represented by a single ARB. The AINE can clearly show the relationships among nodes in its topological network structure.

The AINE has been investigated on the well-known Fisher's Iris data [14]. After 20 iterations, the Setosa class completely disappears from the network representation. Compared with other conventional data mining techniques, we observe that the Kohonen Self-Organizing Map (SOM) [15] can separate the Setosa class and some of the Virginia class. However, the clusters may include both the Virginia and Versicolor classes. The two main parameters, learning rate and neighborhood function, are altered to help spread these clusters out, but they do little in providing adequate separation of the clusters of the Virginia and Versicolor.

In [14], the minimum distance clustering method is applied for the Fisher's Iris data as well. There are two major clusters generated by this technique, one of which describes the Setosa class, and the other is for both the Versicolor and Virginia. These two classes are eventually split after a number of reductions. Reference [7] discusses a new approach for gene expression data clustering based on the aiNet. It is well known that the challenging problems in bioinformatics are generally characterized by very large sets of multivariate data, which present high levels of redundancy and noisy patterns. Reference [16] presents an adaptive unsupervised learning algorithm for generating the AIS. The algorithm introduces the culmination of a series of attempts to obtain a self-organizing meta-stable artificial immune network .Reference [29] proposes that the aiNet, an AIS algorithm exploiting the biologically-inspired features of the immune system, works well on elementary clustering tasks. Based on the immune network and affinity maturation principles, the aiNet performs an evolutionary process on the raw data, which removes data redundancy and retrieves good clustering results.

To summarize, because of the intrinsic features of the AIS, such as nonlinear adaptation and robustness to noise, the methods based on the AIS can identify not only the general patterns in training data, but also the strongest ones. This provides a good opportunity for exploring the AIS as powerful machine learning techniques. However, it is difficult to obtain a clear understanding of the relationship between the individual items in the training data and information extracted from the AINE.

\section{B. Associative classification}

In [23], the AIS is investigated for mining association rules for the Associative Classification (AC). The AC takes the advantage from association rule mining in extracting high quality rules that can accurately generalize the training data set. The AIS-AC proposed in [23] avoids searching greedily for all the possible association rules, and is able to find an effective set of associative rules for classification.

We treat the rule mining process as an optimization problem of finding an optimal set of association rules according to some predefined constraints. The proposed AIS-AC approach is efficient in dealing with the complexity problem in the large search space of rules. The AIS-AC is implemented as follows: Firstly, in each generation, the support constraint is used to filter out specific rules from the population. Next, the confidence values of the rules are deployed for affinity selection. The population is cloned, mutated and diversified. Finally, the best rules in the population are moved to the memory based on the confidence constraint. The process will be terminated when the coverage constraint is satisfied or the number of generations reaches a predefined maximum number of generations.

From Table 1 [23], we can observe that the number of rules used for classification in the AIS-AC is much smaller than that in the AC approach.

\begin{tabular}{rccc}
\hline \multirow{2}{*}{$\begin{array}{r}\text { Support } \\
\text { threshold }\end{array}$} & AIS-AC & \multicolumn{2}{c}{ AC } \\
\cline { 2 - 4 } & No. of rules & No. of frequent sets & No. of rules \\
\hline $10.0 \%$ & 138 & 10794 & 174 \\
$5.0 \%$ & 227 & 34344 & 575 \\
$2.5 \%$ & 202 & 102722 & 1333 \\
$1.2 \%$ & 290 & 280452 & 2689 \\
$0.6 \%$ & 384 & 663276 & 4690 \\
$0.3 \%$ & 327 & 1462940 & 7737 \\
\hline
\end{tabular}

Table 1. Numbers of rules and frequent item sets for Adult data set

Adult is one of the four databases used in the simulations of [23]. Figure 6 shows that the AC algorithm can obtain the complete set of association rules, but the AIS-AC has surprisingly outperformed the $\mathrm{AC}$ with most of the threshold values.

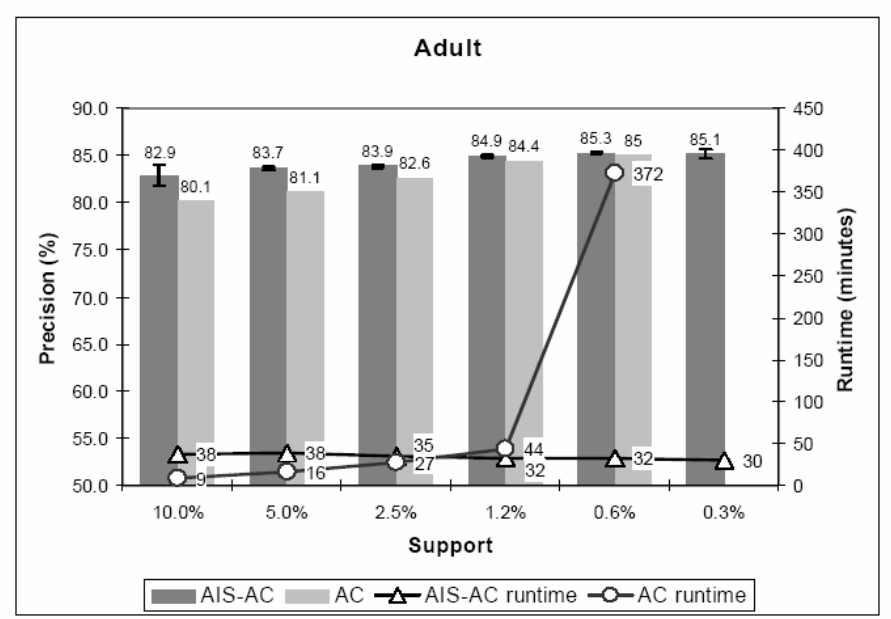

Figure 6. Associative classification performance of Adult data set

The AIS-AC can be also used for mining fuzzy classification rules [24]. In order to cope with continuous attributes, fuzzy logic is employed here. Furthermore, fuzzy logic is a powerful and flexible method to handle uncertainty and improve the rule comprehensibility. The strategy in this study is 
different from the 'divide-and-conquer' and 'separate-and-conquer' approaches in the decision trees and lists, respectively. It actually applies a boosting mechanism to adapt the distribution of training instances in iterations. The AIS can deal with the previously misclassified or uncovered instances, and the cooperation among fuzzy rules is implicitly promoted.

\section{Pattern recognition}

Reference [17] proposes a new artificial immune network with diversity based on the ideas borrowed from the living body immune system, whose dynamics are examined via computer simulations on alphabet pattern recognition. The memory patterns in this system are classified into three unique types, as given in Figure 7. Pattern recognition is performed using the artificial immune network with these memory patterns of such three types. More precisely, Type 1: in a certain fixed preprocessing period, the input pattern becomes the memory pattern. Here, the memory pattern does not update, and, therefore, is retained in the present state. Type 2: when the input pattern has been recognized to be a memory pattern belonging to Type 1, the corresponding memory pattern in Type 2 is updated with the memory pattern and input pattern of the recognized Type 1. Type 3: the memory pattern of this type has the similarity between the updated memory pattern in Type 2 and original memory pattern (memory pattern in Type 1) of $\mathrm{M} \%$ or above. Moreover, the memory pattern recognized and memorized as an unknown pattern also belongs to Type 3 . Note that, in Type 3, the memory pattern, which is not continuously updated for L times, will be deleted.

Reference [13] investigates the application of the above artificial immune network in the pattern recognition system for alphabets. The rate of correct recognition, rate of incorrect recognition, and rate of rejection are employed to evaluate the system performance. Furthermore, the proposed artificial immune network is compared with the binary immune network in order to access its noise tolerance and recognition capabilities, when presented to the binary noise. Simulation results show that with the network diversity, it can acquire stronger noise immunity than the binary immune network for binary input patterns.

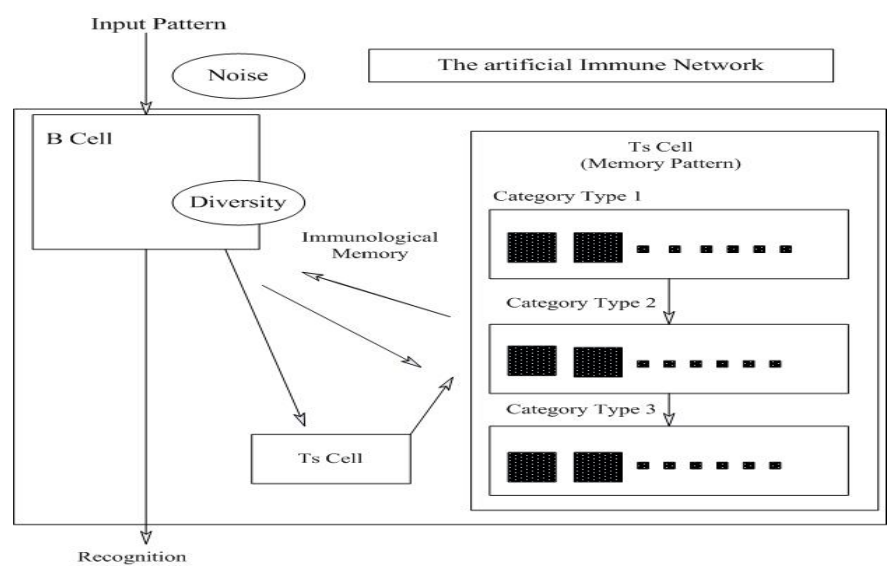

Figure 7. Pattern recognition using artificial immune network

Reference [27] proposes a novel clonal selection method-based artificial immune system. In the mutation process, we select all the mutated antibodies, which have higher affinities than the current memory cell, and regenerate a new candidate memory cell. Simulation results demonstrate that the proposed algorithm has a more effective mutation performance than the CLONALG. A comparison is also made by applying the noisy pattern recognition problem between this method and other AIS models. Recognition results show that this approach has stronger noise immunity and can recognize the unseen noisy patterns more effectively. Figure 8 illustrates the recognition performances of the proposed algorithm and AIS model with 30 generations. Obviously, it is less sensitive to the noise and more efficient in noisy patterns recognition than the AIS model.

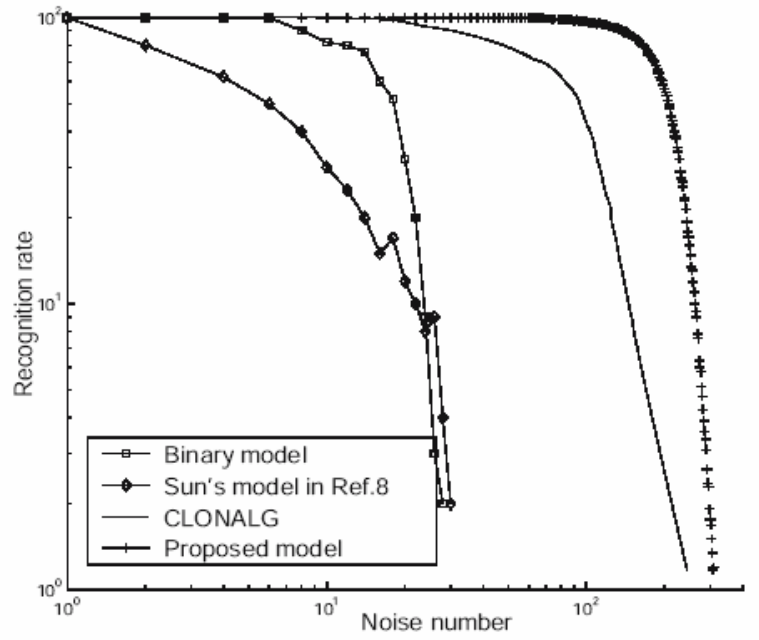

Figure 8. Recognition results of different AIS models

\section{Multimodal function optimization}

Optimization is a popular application area for the artificial immune networks. Reference [18] presents a modified version of the artificial immune network model specially designed to cope with multimodal optimization problems. It is theoretically compared with the clonal selection algorithm concerning their evolution strategies. A new artificial immune optimization method, named CLONALG, is developed in [19] to perform pattern recognition and engineering optimization. The authors empirically demonstrate that the CLONALG is capable of learning the input patterns by selecting, reproducing, and mutating a set of 'artificial immune cells'.

The aforementioned aiNet combining the CLONALG with the artificial immune network theory [20] has been successfully applied in several data compression and clustering applications [12] including non-linear separable and high-dimensional problems. The same rationales that lead to the CLONALG are the motivations for the optimization version of the aiNet, namely opt-aiNet. Firstly, data clustering can be considered as an optimization problem, where each cluster corresponds to the fitness peak of a subgroup of individuals within the whole population. Secondly, the aiNet is an extension of the CLONALG with additional steps involving the stimulation/suppression interaction of the network cells with each other. The advantage of evaluating the degree of similarity among the cells is that it is possible to maintain a dynamic control of the number of network cells, which allows the ultimate finding of more parsimonious solutions. 
The opt-aiNet has been deployed to optimize several uniand bi-dimensional functions to assess its optimization performance. The results are also compared with those obtained by the CLONALG. In [21], three nonlinear functions are explored: multi, roots, and Schaffer's. Figure 9 illustrates the performances of both the CLONALG and opt-aiNet, when employed to the same multimodal function optimization problem. Apparently, the opt-aiNet locates 61 peaks, while the CLONALG locates only 18. Moreover, the opt-aiNet positions one single individual in each peak, which can overcome the harmful 'waste of resources' shortcoming of the CLONALG.

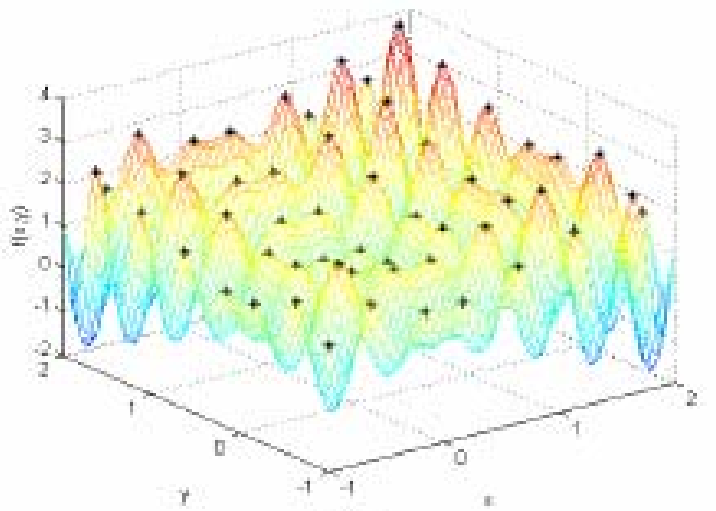

(a)

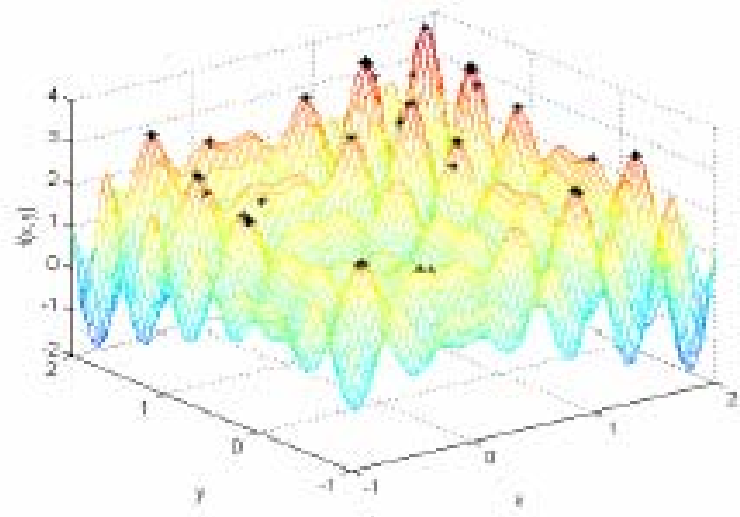

(b)

Figure 9. Multimodal function optimization (a) using opt-aiNet. (b) using CLONALG

\section{E. Others Applications}

A novel artificial immune system-based data storage model, called AIS-DS, is proposed for dealing with the problem of resources sharing in a Storage Area Network (SAN) [28]. Especially, for the multi-user's tasks, this technology has some essential features for ensuring the security and privacy of information and/or data. An extended examination of the spam-detection artificial immune system is given in [36]. The system can distinguish between a self of legitimate email (non-spam) and a non-self of spam.

\section{F. Experiments}

\section{1) Clustering algorithms}

Clustering is an unsupervised knowledge discovery process that groups a set of data such that the intra-cluster similarity is maximized while the inter-cluster similarity is minimized. We here discuss a novel clustering algorithm called Fuzzy Artificial Immune System Clustering (FAISC) [38], which is based on the artificial immune network [37] and fuzzy system. More precisely, at the first beginning of training, we initialize the AIS network with several data items and assign one fixed value. We add a parameter CHANGE to the original system [5], and the pattern of the ARB can be calculated as follows:

$$
\text { Pattern }_{\text {new }}=\frac{\text { Pattern }_{\text {new }} \times \text { Change }+ \text { Pattern }_{\text {old }}}{1+\text { Change }},
$$

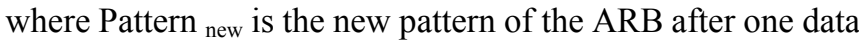
item enters the network, and Pattern old is the old pattern of ARB before that data item comes.

For evaluating a single clustering, we use totally three measurements, i.e., purity, balance, and f1. We compare the FAISC with the K-means method on the well-known iris data set, and we observe that if the value of the NAT is fixed to be 1.2 or 1.0 , the result of the FAISC is obviously better than that of the k-means approach. Especially, when the NAT is 1.2, the corresponding result is the best.

\section{2) Classification algorithms}

In our paper [39], we propose an AIS-based scheme for software quality classification. We also compare this method with other well-known classification techniques: Naive Bays, Nearest Neighbor, and OneR. The simulation results demonstrate that our AIR is a promising method for software quality classification.

\section{Remarks and Conclusions}

The success of an AIS practitioner owes much to the theory presented by the immunologists. It is clear from many examples of the AIS that the way in which they are designed has changed from the early days of the AIS research. The original AIS, such as those proposed by Bersini [10], Forrest et al. [11], and Hightower et al. [12], are developed using an interdisciplinary approach with clear inspiration from the biology. More recently, however, the design focus of the AIS has become more engineering oriented, with less emphasis on understanding and extracting the key biological models [25].

In this paper, we give an overview of the recently proposed artificial immune networks. The structures and learning algorithms of a few typical artificial immune networks are discussed. We also demonstrate how they can be employed in dealing with real-world problems of data mining, pattern recognition, associative classification, and multimodal function optimization. Although they have achieved great successes in various engineering areas, there are still some theoretical issues that need to be further explored, e.g., convergence and running stability. The developments of the artificial immune networks would benefit from not only the inspiration of natural immune principles, but also merging with other soft computing paradigms, such as neural networks, fuzzy logic, and genetic algorithms. In addition, they could be generalized to more challenging application areas.

\section{Acknowledgments}

The work was supported by the National Science Foundation of China under the Grant No. 10001006 and No. 60273015. X. Z. Gao's work was funded by the Academy of Finland under 
Grant 214144. The authors would like to thank the anonymous reviewers and chief editor for their insightful comments and constructive suggestions that have improved this paper.

\section{References}

[1] J. Timmis and T. Knight, Artificial immune systems: Using the immune system as inspiration for data mining. In Data Mining: A Heuristic Approach, H. A. Abbass, R. A. Saker, and C. S. Newton (eds.): Idea Group Publishing, pp. 209-320 (2001).

[2] J. Timmis and M. Neal, A resource limited artificial immune system for data analysis. Knowledge-Based System 14, pp. 121-130 (2001).

[3] J. Suzuki and Y. Yamamoto, iNet: An extensible framework for simulating immune network. In Proc. of IEEE International Conference on Systems, Man, and Cybernetics, pp. 119-124 (2000).

[4] J. Timmis, M. Neal, and J. Hunt, An artificial immune system for data analysis. BioSystems 55, pp.143-150 (2000).

[5] J. Timmis, Artificial immune systems: a novel data analysis technique inspired by the immune network theory. Ph.D. Thesis, University of Wales, Aberystwyth, UK (2000).

[6] M. Neal, An artificial immune system for continuous analysis of time-varying data. In Proc. of ICARIS, pp. 76-85 (2002).

[7] G. B. Bezerra and L. N. de Castro, Bioinformatics data analysis using an artificial immune network. In Proc. of ICARIS. LNCS 2787, pp. 22-33 (2003).

[8] L. N. de Castro and F. J. von Zuben, An evolutionary immune network for data clustering. IEEE SBRN, pp. 84-89 (2000).

[9] S. Sahan, H. Kodaz, S. Gunes and K. Polat, A new classifier based on Attribute Weighted Artificial Immune System (AWAIS). In Proc. of ISCIS, LNCS 3280, pp. 11-20 (2004).

[10] R. Romis, F. Attux, L. N. de Castro, F. J. von Zuben, and J. T. Marcos, A paradigm for blind IIR equalization using the constant modulus criterion and an artificial immune network. In Proc. of IEEE XIII Workshop on Neural Networks for Signal Processing, pp. 839-848 (2003).

[11] J. Hunt, J. Timmis, D. Cooke, M. Neal, and C. King, JISYS: the development of an artificial immune system for real world applications. Applications of Artificial Immune Systems, D. Dasgupta (ed.), pp. 157-186, Springer-Verlag, (1999).

[12] M. Neal, J. Hunt, and J. Timmis, Augmenting an artificial immune network. In Proc. of Int. Conf. on Systems and Man and Cybernetics, pp. 3821-3826 (1998).

[13] T. Knight and J. Timmis, AINE: An immunological approach to data mining. In Proc. of IEEE International Conference on Data Mining, pp. 297-304 (2001).

[14] R. A. Fisher, The use of multiple measurements in taxonomic problems, Annals of Eugenics 7, pp. 179-188, (1936).

[15] T. Kohonen, Self-Organizing Maps. Berlin, Germany: Springer-Verlag, 1995.

[16] M. J. Neal, Meta-stable memory in an artificial immune network. In Proc. of the International Conference on
Artificial Immune Systems, Edinburgh, UK, LNCS 2787, pp. 168-180. Springer (2003).

[17] W.-D. Sun, X.-S. Xu, H.-W. Dai, Z. Tang, and H. Tamura, An artificial immune network with diversity for pattern recognition. In Proc. of the SICE Annual Conference in Sapporo August (2004).

[18] L. N. de Castro and J. Timmis, An artificial immune network for multimodal function optimization, In Proc. of IEEE CEC, pp. 699-674 (2002).

[19] L. N. de Castro and F. J. von Zuben, Learning and optimization using the clonal selection principle, IEEE Transactions on Evolutionary Computation (2001).

[20] N. K. Jerne, Towards a network theory of the immune system, Annual Immunology. (Inst. Pasteur) 125C, pp. 373-389 (1974).

[21] L. N. de Castro and F. J. von Zuben, aiNET: An artificial immune network for data analysis. In Data Mining: A Heuristic Approach, H. A. Abbass, R. A. Saker, and C. S. Newton (eds.), Idea Group Publishing, Chapter XII, pp. 231-259.

[22] M. Negoita, Artificial immune systems-An emergent technology for autonomous intelligent systems and data mining. AIS-ADM 2005, LNAI3505, pp. 19-36, 2005.

[23] T. D. Do, S. C. Hui, and A. C. M. Fong, Artificial immune system for associative classification. ICNC 2005, LNCS 3611, pp. 849-858, 2005.

[24] B. Alatas and E. Akin. Mining fuzzy classification rules using an artificial immune system with boosting. ADBIS 2005, LNCS 3631, pp. 283-293, 2005

[25] P. S. Andrews and J. Timmis. Inspiration for the next generation of artificial immune systems. LNCS 3627, pp. 126-138, 2005.

[26] Z. Wu and Y. Liang. Self-regulation methods for model library based artificial immune systems. LNCS 3627, pp. 353-365, 2005.

[27] H. Dai, Y. Yang, Y. Che, and Z. Tang. Clonal selection theory based artificial immune system and its application. LNCS 4233, pp. 1071-1078, 2006

[28] L. Wang, Y. Nie, W. Nie, and L. Jiao. Artificial immune strategies improve the security of data storage. ICNC 2005. LNCS 3611, pp. 839-848, 2005.

[29] N. Tang and V. R. Vemuri. An artificial immune system approach to document clustering. SAC'05. March 2005

[30] D. Dasgupta, K. KrishnaKumar, D. Wong, and M. Berry, Negative selection algorithm for aircraft fault detection. In Proc. of the Third International Conference on Artificial Immune Systems, Catania, Sicily, Italy, Sept. 2004

[31] J. Kim, W. O. Wilson, U. Aickelin, and J. McLeod. Cooperative Automated Worm Response and Detection ImmuNe ALgorithm (CARDINAL) inspired by T-cell immunity and tolerance. In Proc. of the Fourth International Conference on Artificial Immune Systems, Banff, Alberta, Canada. pp. 168-181, Aug. 2005.

[32] J. Timmis, M. Neal, and T. Knight. AINE: Machine learning inspired by the immune system. IEEE Transactions on Evolutionary Computation, June 2002.

[33] Y. Cao and D. Dasgupta, An immunogenetic approach in chemical spectrum recognition. Chapter 36 in Advances in Evolutionary Computing (Ghosh \& Tsutsui, eds.), Springer-Verlag, Inc. Jan. 2003.

[34] A. Iqbal and M. A. Maarof, Towards danger theory based artificial APC model: Novel metaphor for danger susceptible data codons. In Proc. of the Third Interna- 
tional Conference on Artificial Immune Systems, pp. 161-174, 2004.

[35] S. Stepney, R. E. Smith, J. Timmis et al. Towards a conceptual framework for artificial immune systems. In Proc. of the Third International Conference on Artificial Immune Systems, pp. 53-64, 2004.

[36] T. Oda and T. White. Immunity from spam: An analysis of an artificial immune system for junk email detection. In Proc. of the Fourth International Conference on Artificial Immune Systems, Banff, Alberta, Canada, pp. 276-289, Aug. 2005.

[37] M. Neal. Meta-stable memory in an artificial immune network. In Proc. of the 2nd International Conference on Artificial Immune Systems Springer, pp.168-180, 2003

[38] Z. Liu, X. Jin, R. Bie, and X. Z. Gao. FAISC: a Fuzzy Artificial Immune System Clustering Algorithm. In Proc. of the Third International Conference on Natural Computation, pp. 657-661, 2007.

[39] X. Jin, R. Bie, and X. Z. Gao. An artificial immune recognition system-based approach to software engineering management: with software metrics selection. In Proc. of the Sixth International Conference on Intelligent System Design and Applications, pp. 523-528, 2006. 